\title{
Evaluation of hypoglycemic effect of compound(s) from petroleum ether fraction of ethanol extract of Mangifera indica red leaves
}

\author{
Abdul Alim ${ }^{1}$ Rokshana Sharmin ${ }^{2}$, Md. Sarowar Hossain ${ }^{3}$, Debendra Nath Ray ${ }^{2}$, \\ Md. Rafiqul Islam khan ${ }^{4 *}$, Md. Ariful Islam ${ }^{4}$, Most. Afia Akhtar ${ }^{4}$, Abu Syed \\ Md. Anisuzzaman ${ }^{4}$, Maruf Ahmed ${ }^{4}$ \\ ${ }^{1}$ Square Pharmaceuticals Ltd., Kaliakoir, Gazipur-1750, Dhaka, Bangladesh. \\ ${ }^{2}$ Department of Pharmacy, Jessore Science and Technology University, Jessore-7408, Bangladesh. \\ ${ }^{3}$ Department of Pharmacy, Atish Dipankar University of Science and Technology., Dhaka, Bangladesh. \\ ${ }^{4}$ Department of Pharmacy, Rajshahi University, Rajshahi-6505, Bangladesh.
}

\begin{abstract}
The petroleum ether fraction of ethanol extract of Mangifera indica red leaves decreased fasting blood glucose (FBG) level of approx. $90 \%$ in alloxan induced diabetes rats (AIDRs). However, the specific compound(s) has not been clarified. The present study has been designed to separate hypoglycemic compound(s) using preparative thin layer chromatography (PTLC) from petroleum ether fraction of ethanol extract of Mangifera indica red leaves. Development of TLC demonstrated the presence of distinct three layers having the $R_{\mathrm{f}}$ values $0.86,0.56$ and 0.28 on solvent system, chloroform: $n$ hexane (80:20). Compounds with $R_{f}$ values 0.86 and 0.56 reduced fasting blood glucose level by 92 and 96\%, respectively in AIDRs. However, the compound with $R_{f}$ value 0.28 did not have any significant antihyperglycemic effect. It was found from the experiment that the compound with $R_{f}$ value 0.56 had predominant hypoglycemic effect than the other. Therefore, it can be a potential candidate for screening the lead for newer antidiabetic drugs that can offer a natural key to manage diabetes for the future.
\end{abstract}

Keywords: - Alloxan, hypoglycemic, PTLC, TLC.

\section{INTRODUCTION}

Diabetes is a global problem and number of those affected is increasing day by day. It is affecting nearly $10 \%$ of the population worldwide. ${ }^{[1]}$ Diabetes mellitus is a metabolic disorder characterized by chronic hyperglycemia resulting from defects in insulin secretion, insulin action, or both. It is associated with reduced life expectancy, significant morbidity due to specific diabetes related microvascular complications (retinopathy, nephropathy and neuropathy), increased risk of macrovascular complications (ischemic heart disease, stroke and peripheral vascular disease) and diminished quality of life. ${ }^{[2]}$ The major mode of controlling diabetes can be achieved by diet, exercise, and insulin replacement therapy and/or by different oral hypoglycemic drugs. However, treatment with sulfonylureas and biguanides is associated with side effects and fail to alter the course of diabetic complications significantly. ${ }^{[3]}$ In modern medical system, managing diabetes without side effects is still a challenge. The search for new pharmacologically active agents obtained by screening natural sources such as medicinal plants or their extracts has led to the discovery of many clinically useful drugs that play a major role in the treatment of human diseases. ${ }^{[4]}$

Now a days, scientists and researchers are very much interested on research of natural plant products all over the world and a large amount of substantiation have shown the immense potential of medicinal plants used traditionally. ${ }^{[5]}$ In the last few years there has been an exponential growth in the field of herbal medicine and these drugs are gaining popularity both in developing and developed countries. ${ }^{[6]}$ However, it should be kept in mind that phytotherapy is not an alternative but a complementary and supportive treatment to the conventional diabetes therapy. One reason may be due an inappropriate dosage regimens and inadequate supply of the exact compounds exists in the herbs. Therefore, a proper scientific evaluation of the plant by pharmacological tests followed by chemical investigations is necessary for the invention of active chemicals or leads for the development of new potential antidiabetic drugs. One of the most prominent antidiabetic drug metformin, already leading in the diabetologist's pharmacy is also generated from a plant originated from French Lilac (Galega officinalis) that is using successfully in diabetes treatment for the last five decades.

In the light of the literature, experimental animal study by the stem-bark aqueous extract of Mangifera indica Linn. suggested the management of adult-onset type 2 diabetes mellitus in some rural African communities. ${ }^{[7]}$ We have previously reported the antidiabetic effects of various fractions (Petroleum ether, ethyl 
Evaluation of hypoglycemic effect of compound(s) from petroleum ether fraction...

acetate and chloroform) of the ethanol extract of Mangifera indica red leaves in AIDRs. ${ }^{[8]}$ In the present work, we have attempted to find out hypoglycemic compound(s) from the petroleum ether fraction of Mangifera indica red leaves. PTLC plates were used for the collection of compound(s) and alloxan-induced diabetic rats were used for the evaluation of antidiabetic effects.

\subsection{Drugs and Chemicals}

\section{MATERIALS AND METHODS}

The standard antidiabetic agent, metformin hydrochloride was the generous gift samples from Square Pharmaceuticals Ltd., Pabna Bangladesh. Alloxan was purchased from Sisco Research Laboratories Ltd., Mumbai, India.

\subsection{Plant materials}

The fresh leaves of Mangifera indica (Local name- Aam) were collected from medicinal plant garden at Naogaon, Bangladesh. These were dried completely under the mild sun and ground with an electric grinder into coarse powder and used for cold extraction.

\subsection{Preparation and fractionation of crude extracts}

The coarse powder was submerged in ethanol (96\%) and allowed to stand for several days (7-10) with occasional shaking and stirring. When the solvent become concentrated, the liquid alcohol content was filtered through cotton and then through filter paper (Whatman filter paper no. 1). Then the solvents were allowed to evaporate using rotary evaporator at $40-45^{\circ} \mathrm{C}$ and the highly concentrated crude extracts were obtained. They were then fractionated using petroleum ether. The dried fractionated extracts were then preserved at $4^{\circ} \mathrm{C}$ for the experimental use. ${ }^{[9]}$

\subsection{Preparation of TLC and PTLC plates}

One dimensional thin layer chromatographic (TLC) technique was used for the initial screening of compounds from chloroform and petroleum ether fraction. TLC and PTLC plates were made on glass plates (20 $\mathrm{cm} \mathrm{X} 5 \mathrm{~cm}$ for TLC and $20 \mathrm{~cm} \mathrm{X} 20 \mathrm{~cm}$ for PTLC) with silica gel (Kiesel gel 60 PF 254). These plates were thoroughly washed and dried in an oven. These dried plates were then swabbed with acetone to remove any fatty residue. To make the slurry silica gel $60 \mathrm{PF}-254$ and appropriate volume of distilled water $(2 \mathrm{ml} / \mathrm{gm}$ of silica for TLC and $3 \mathrm{gm}$ silica/ plate for PTLC) were mixed in a conical flask and the flask was gently shaken. Slurry was then evenly distributed over the plates using TLC spreader. After air-drying the coated plates were subjected to activation by heating in an oven at $110^{\circ} \mathrm{C}$ for 70 minutes.

\subsection{Development of TLC plates}

Using trial and error assumption different solvents in different ratios were tried. TLC tank with airtight lid was used for the development of chromatoplates. Filter paper was introduced into the tank and allowed to soak in the solvent. Then the tank was kept airtight for few minutes to saturate the internal atmosphere with the solvent vapor. A small amount of dried extracts (chloroform and petroleum ether fractions) were dissolved in a suitable solvent to get a solution. A vertical line was drawn in the middle position on it and spotted on the plate with capillary tube just $1 \mathrm{~cm}$ above the lower edge of the plate. Then it was dried and drawn a straight-line $2 \mathrm{~cm}$ below from the upper edge of the plate. Spotted plate was then placed in the TLC tank contain solvents with selected proportion and allowed it for developing. Running the solvent(s) to the marked upper layer it was taken out and air-dried. The compounds were then detected from the developed plates. The following techniques were used to detect the compounds in TLC plates; Visual detection, UV- light, Iodine chamber. $\mathrm{R}_{\mathrm{f}}$ value of the compounds were calculated by the following equation:

$\mathrm{R}_{\mathrm{f}}$ Value $=\frac{\text { Distance traveled by the compound }}{\text { Distance traveled by the solvent system }}$

\subsection{Experimental design on Rat model}

Total 30 rats were used in this study and divided into six groups. Rats were collected from ICCDR, Bangladesh. Each group contains 5 rats. After induction of chemical diabetes (group II to group IV), group II was selected for diabetic control, which did not receive either metformin, or plant extracts. Group III was for metformin control in which metformin was administered intraperitoneally at a dose of $150 \mathrm{mg} / \mathrm{kg}$ body weights. Group IV, V and VI received plant extracts with $\mathrm{R}_{\mathrm{f}}$ values $0.28,0.56$ and 0.86 layer containing compound(s), respectively. Group I was normal control group, also did not receive either metformin or plant extracts. The plant extracts were administered intraperitoneally at a same dose of metformin standard. The blood samples 
Evaluation of hypoglycemic effect of compound(s) from petroleum ether fraction...

were analyzed for blood glucose content at different time points as indicated in figures. Blood glucose level was estimated in all the experiments by using glucometer (Bioland-423, Germany).

\subsection{Statistical Analysis:}

Data were expressed as mean \pm standard error of mean (SEM). Statistical comparisons were performed by one-way analysis of variance (ANOVA), or students paired or unpaired $t$-test where appropriate. Results were considered to be significant when $p$ values were less than $0.05(p<0.05)$. Statistical calculations and the graphs were prepared using Graph Pad Prism version 5.00 for Windows (Graph Pad Software, San Diego, CA, USA).

\section{RESULTS}

\subsection{Antihyperglycemic effects of petroleum ether fraction on AIDRs}

Petroleum ether fraction of ethanol extract reduced fasting blood glucose level to $70.21 \%, 58.26 \%$, and $13.64 \%$ in 2, 6, and $16 \mathrm{hrs}$, respectively. Maximum reduction of blood glucose level by $86.36 \%$ was observed in $16 \mathrm{hr}$ time points during the experimental period.

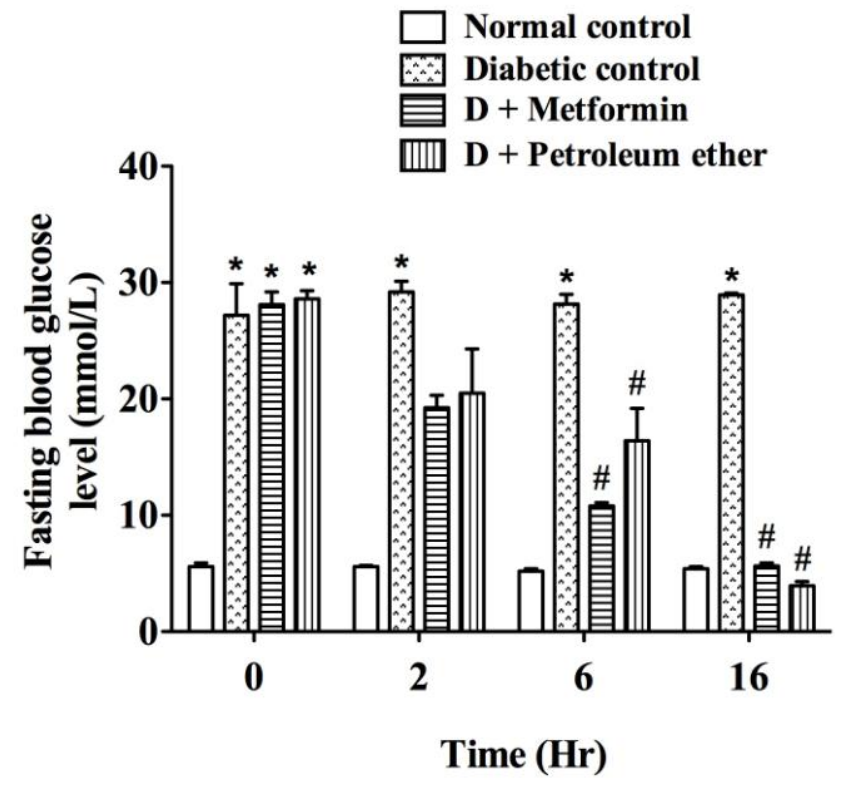

Fig. 1 Effect of petroleum ether fraction of ethanol extract on the FBG level in diabetic rats compared to normal rats. * indicates significant changes (increase) of blood glucose level compared with normal control group. \# indicates significant changes (decrease) of FBG level in diabetic rats after treatment compared with zero hr treatment group. The results are expressed as means \pm SEM.

\subsection{Isolation of compounds from the petroleum ether fraction of Mangifera indica}

TLC plates were used to detect different compounds from the petroleum ether fraction of Mangifera indica ethanol extract using chloroform and $n$-hexane in the ratio 80:20. Three layers were detected with $R_{\mathrm{f}}$ values $0.86,0.56$ and 0.28 as shown in fig. 2 (A, B). Compounds with $R_{f}$ values 0.86 and 0.56 were visually detected on developed TLC plate but that of $R_{f} 0.28$ only responded under the UV light and Iodine chamber. In iodine chamber it absorbed iodine and became brown. The three separated layers were scraped (compounds with silica gel) from PTLC plate and collected in closed bottle $\left(\mathrm{R}_{\mathrm{f}} 0.28\right.$; prevent oxidation) and open beakers $\left(\mathrm{R}_{\mathrm{f}} 0.86\right.$ and 0.56), respectively. Methanol and ethyl acetate solvent at a ratio of 3:7 were used to separate compounds from silica gel. Then these solvents were evaporated to get solid mass. Approximately 200 PTLC plates were used to get sufficient amount of yields. Total yield was $400 \mathrm{mg}$ approximately. These solid masses were dissolved in dimethyl sulfoxide (DMSO) solution and applied to rat model for the evaluation of antihyperglycemic effects. 


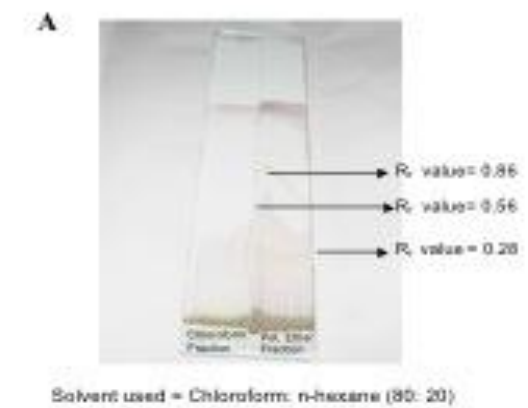

TLC developed by using chloroform and n-hexane at a ratio of $80: 20$
B

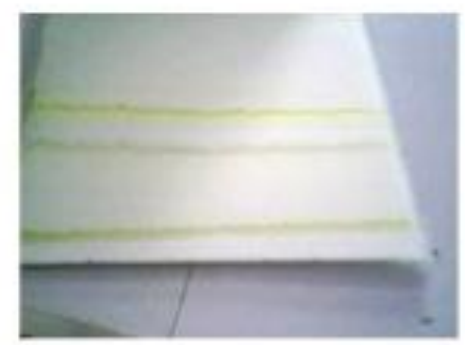

PTLC was used to elute separate bands

Fig. 2 Petroleum ether fraction shows three distinct layers on the TLC plate using the solvent system chloroform: $n$-hexane (80:20) with $R_{f}$ values $0.86,0.56$, and 0.28 (A). Compounds with $R_{f}$ values 0.86 and 0.56 were visually detected on developed TLC plate but that of $\mathrm{R}_{\mathrm{f}} 0.28$ only responded under the UV light and Iodine chamber (B).

\subsection{Antidiabetic effects of the separated crude extracts on rat model}

The parameters of fasting blood glucose (FBG) level was measured to search for the antidiabetic compound (s) from petroleum ether fraction of the ethanolic extracts of Mangifera indica on normal and alloxan induced diabetic rats using metformin as standard antidiabetic agent. The effects of metformin and different compounds separated form petroleum ether fraction of Mangifera indica on FBG level in normal and alloxan induced diabetic rats are shown in the fig. 3. The significant decrease of FBG observed from $4 \mathrm{hr}$ after treatment and sustained the reduction until $16 \mathrm{hr}$. Maximum reduction of FBG level was $95.5 \%$ and $92 \%$ for the layer $R_{f} 0.56$ and $R_{f} 0.86$, respectively at $16 \mathrm{hr}$ of the experimental period. However, the compound for the layer Rf 0.28 was ineffective all over the treatment period.

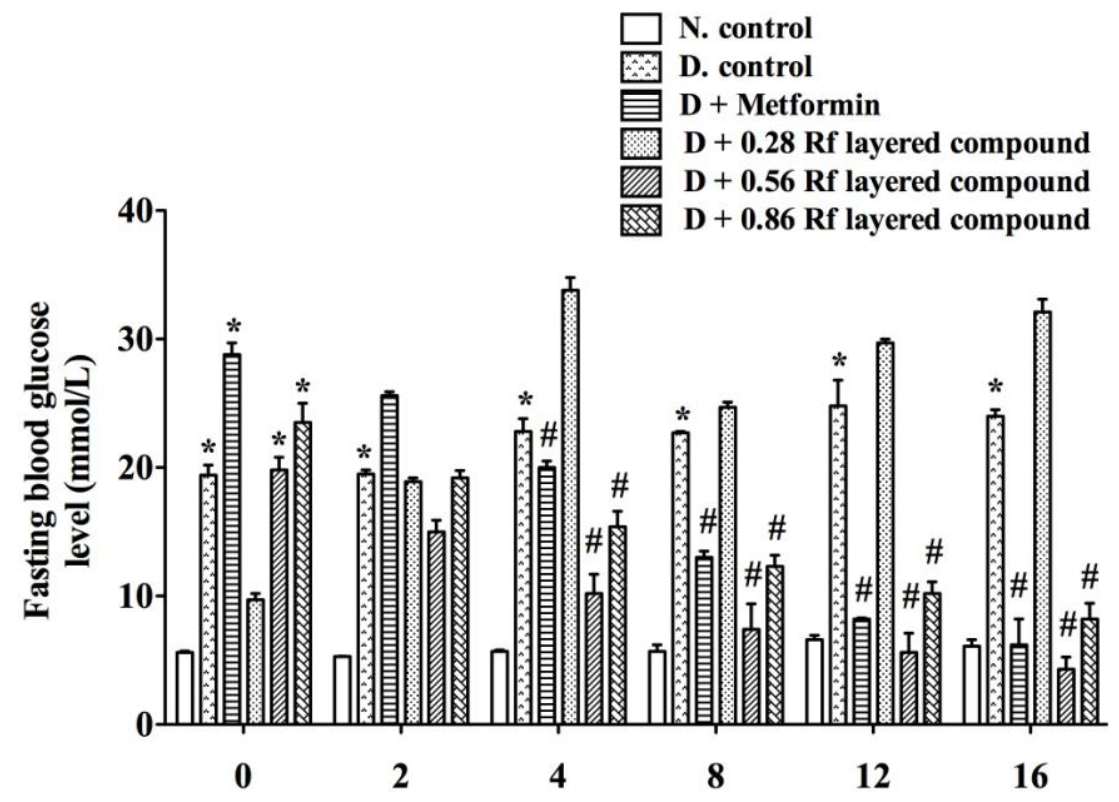

Fig. 3 Effect of different separated compounds on the FBG level on diabetic rats compared to normal rats. * indicates significant changes (increase) of blood glucose level compared with normal control group. \# indicates significant changes (decrease) of FBG level in diabetic rats after treatment compared with zero hour treatment group. The results are expressed as means \pm SEM.

\section{DISCUSSION}

Numerous oral hypoglycemic drugs exist alongside insulin; still there is no promising therapy to cure diabetes. ${ }^{[10]}$ In recent years, many traditional medicinal plants were tested for their antidiabetic potential in the experimental animals. ${ }^{[11]}$ In the light of the literature on Mangifera indica we made an attempt for the first time 
Evaluation of hypoglycemic effect of compound(s) from petroleum ether fraction...

to isolate compounds from petroleum ether fraction of Mangifera indica ethanolic extract and to study the effect of specific compound(s) of partitionates in alloxan induced hyperglycemic rats.

We have isolated three layers of compounds with $\mathrm{R}_{\mathrm{f}}$ values $0.86,0.56$ and 0.28 (Fig. 2). Among them compound $\left(\mathrm{R}_{\mathrm{f}}\right.$ 0.56) reduced FBG of $95.5 \%$ and compound $\left(\mathrm{R}_{\mathrm{f}}\right.$ value 0.86$)$ reduced FBG of $92 \%$ in alloxan induced diabetic rats (AIDR). The significant antidiabetic activity of compounds $\left(R_{f}\right.$ value 0.56$)$ and $\left(R_{f}\right.$ value 0.86) of petroleum ether fraction of ethanol extract of Mangifera indica has been shown in Fig. 3.

In this study, the antihyperglycemic action of the Mangifera indica was better (especially $\mathrm{R}_{\mathrm{f}} 0.56$ fraction) than that of the standard drug, metformin. The possible mechanism by which Mangifera indica brings about its antidiabetic action may be by potentiating the insulin effect of plasma by stimulating insulin release from the remnant pancreatic $\beta$-cells or its release from the bound form. ${ }^{[12]}$ Beside this, it might involve in extrapancreatic action in these alloxan-diabetic rats, which might include the stimulation of peripheral glucose utilization or enhancing glycolytic and glycogenic processes with concomitant decrease in glycogenolysis and gluconeogenesis. ${ }^{[13]}$

Lastly, the antihyperglycemic activity of Mangifera indica may be due to the presence of hypoglycemic saponins, tannins, triterpines, alkaloids, flavonoids etc. ${ }^{[7]}$ On the basis of the current investigation it was noted that the compounds $\left(\mathrm{R}_{\mathrm{f}}\right.$ value.56) and $\left(\mathrm{R}_{\mathrm{f}}\right.$ value.86) of petroleum ether fraction of ethanol extract of Mangifera indica acted in a similar fashion to metformin (standard drug) and it can be suggested that these results provide pharmacological evidence for its folklore claim as an anti-diabetic agent.

\section{CONCLUSOINS}

From the current study, it is concluded that the compounds $\left(R_{f}\right.$ value 0.56$)$ and $\left(R_{f}\right.$ value 0.86$)$ of petroleum ether fraction of ethanol extract of Mangifera indica has potent antidiabetic effects in a similar extent as that of metformin. Therefore, we believe that the study of specific hypoglycemic compound(s) from petroleum ether fraction of Mangifera indica ethanol extract offer a natural key to unlock a diabetologist's pharmacy for the future. However, further study is necessary for structure elucidation of the respective antidiabetic compounds.

\section{ACKNOWLEDGEMENTS}

We thank Pharmacy Department of Rajshahi University for providing necessary facilities to carry out this research work. The present work was supported by the National Science and Information and Communication Technology (NSICT), Dhaka, Bangladesh for financial assistance to the second author and the authors would like to extend their gratitude to the Director, Animal Research Centre (ARC), ICDDR, B for providing necessary facilities.

\section{REFERENCES}

1). T. Vetrichelvan, M. Jegadeesan and B.A.V. Devi, Anti-diabetic Activity of Alcoholic Extract of Celosia argentea LINN. Seeds in Rats, Biol Pharm Bull, 25, 2002, 526-528.

2). Definition and diagnosis of diabetes mellitus (WHO and IDF, Geneva, Switzerland, 2006) pp. 5.

3). K.M. Ali, K. Chatterjee, D. De, T. K. Bera and D. Ghosh, Efficacy of aqueous extract of seed of Holarrhena antidysenterica for the management of diabetes in experimental model rat: A correlative study with antihyperlipidemic activity, International J. Applied Res. in Natural Products, 2 (3), 2009, 13.

4). M. Modak, P. Dixit, J. Londhe, S. Ghaskadbi and T. P. A. Devasagayam, Indian Herbs and Herbal Drugs Used for the Treatment of Diabetes, J. Clin. Biochem. Nutr. 40 (3), 2007, 163-173.

5). J.K. Grover, S. Yadav and V. Vats, Medicinal plants of India with anti-diabetic potential. $J$ Ethnopharmacol, 81, 2002, 81-100.

6). P. Hemant, S. Sameer, S.K. Balvant, J. Kusum and J. GC, Evaluation of hypoglycemic and antihyperglycemic potential of Tridaxprocumbens, BMC Complementary and Alternative Medicine, 9, $2009,48$.

7). J.A. Ojewole, Anti-inflammatory, analgesic and hypoglycemic effects of Mangifera indica Linn. (Anacardiaceae) stem-bark aqueous extract, Exp. Clin. Pharmacol, 27(8), 2005, 547-554.

8). M.S. Hossain, M.R.I. Khan, A.S.M. Anisuzzaman, M. Ahmed, M.S. Amran and A. Islam, Antidiabetic and Glycogenesis Effects of Different Fractions of Ethanolic Extract of Leaves of Mangifera indica in Normal and Alloxan Induced Diabetic Rats, Journal of Medical Sciences, 10(4), 2010, 80-86.

9). A.H.M Khurshid Alam, Antishigella activity of Hemigraphishirta T. Anders and AchyranthusferrugineaRoxb. M. Pharm. diss., Department of Pharmacy, University of Rajshahi, Bangladesh, 1999.

10). G. Sumana and S.A. Suryawanshi, Effect of Vinca rosea extracts in treatment of alloxan diabetes in male albino rat, Indian J. Exp. Biol., 39, 2001, 748-759. 
Evaluation of hypoglycemic effect of compound(s) from petroleum ether fraction...

11). Y. Srivastava, H.V. Bhatt, Y. Verma, K. Venkaiah and B.H. Raval, Antidiabetic and adaptogenic properties of Momordica charantia extract, an experimental and clinical evaluation. Phytother. Res., 7, 1993, 285-289.

12). I.M. Mahomed, J.A. Ojewole, Hypoglycemic effect of Hypoxis hemerocallidea corm (African potato) aqueous extract in rats, Exp Clin Pharmacol 25, 2003, 617-623.

13). A. Andrade-Cetto and H. Wiedenfeld, Hypoglycemic effect of Acosmiumpanamense bark on streptozotocin diabetic rats. J Ethnopharmacol 2004, 2004, 217-220. 